\title{
A Review of Cellular Networks: Applications, Benefits and
}

\section{Limitations}

\author{
Adewumi Adebayo Segun ${ }^{1 *}$, Francis Dubem Chizea ${ }^{2}$ and Ayantunji Gbenro Benjamin ${ }^{3}$ \\ ${ }^{1}$ Department of Pure and Applied Physics Ladoke Akintola University of Technology, Ogbomoso, Oyo State \\ Nigeria. \\ ${ }^{2,3}$ National Space Research and Development Agency, Abuja, Nigeria \\ *corresponding author: asadewumi@lautech.edu.ng
}

\begin{abstract}
Over decades the world has witnessed stepwise evolution in Cellular networks technology and mobile network industry which have transformed nation's economy and created job opportunities since 1970. The stepwise evolution of the cellular networks from first generation $(1 \mathrm{G})$ to fifth generation $(5 \mathrm{G})$ have shown tremendous increase in technology, benefits, user demand and applications. As new generation of cellular network unfold, the challenges and limitations of preceded generations are being tackled as always depicted in the design architecture of each new generation. The first generation (1G) cellular network was based on analogue and was able to cater for mobile voice transmission but posed some challenges in terms of quality of service and security of network. Second generation $(2 \mathrm{G})$ came with the introduction of digitally encrypted technology and greater security for sender and receiver with services such as text messages and MMS. Third generation (3G) was developed to offer high speed data and multimedia connections to subscribers. Fourth generation evolves from $3 \mathrm{G}$ with higher data rate, lower latency, greater spectral efficiency and simple protocol architecture with efficient multicast than its predecessors. Fifth-generation $(5 \mathrm{G})$ networks is being deployed to meet growing demands for data from consumer and industrial users and to enable the use of advanced technologies such as smart city applications, autonomous vehicles and navigation. The envisioned sixth generation (6G) of cellular network is expected to witness an unparalleled revolution that would significantly distinguish it from the existing generations and will drastically re-shape the wireless evolution from "connected thing to connected intelligence. This paper provides a comprehensive review of cellular networks applications and challenges from $1 \mathrm{G}$ to $6 \mathrm{G}$.
\end{abstract}

Keywords: $1 G, 2 G, 3 G, 4 G, 5 G, 6 G$, Applications Benefits, and Limitations

DOI: $10.7176 / \mathrm{NCS} / 11-04$

Publication date: December 31st 2020

\subsection{Introduction}

With each passing day, the maturity level of the mobile user and the complexity level of the cellular network reaches a new high. The cellular technology evolution has been going on since the late 1950s, though the first commercial systems came into being in the late 1970s and early 1980s (Mishra R. A; 2007). The development of a truly personal communications space will rely on the design of next generation wireless systems based on a whole new concept of fast, reconfigurable networks, supporting features such as high data rates, user mobility, adaptability to varying network conditions, and integration of a number of wireless access technologies, and offering new user-centric flexible service paradigms (Angeliki and Martin 2004). Cellular technologies and networks have made tremendous impacts on the development and fast evolution of the mobile communications. Cellular networks are no more limited to traditional GSM networks but a complex mixture of generation technologies of cellular networks. Gone are the days of planning GSM, EGPRS or WCDMA networks individually. Now the cellular network business is about dimensioning for new and advanced technologies of cellular networks (Mishra R. A; 2007). 


\subsection{Evolutionary of Cellular Networks}

\subsection{First Generation Cellular Networks (1G)}

Since the late 1970s when the cellular era started, mobile communication has gone through an evolutionary change every decade in terms of technology and usage (Mishra R. A; 2007). Cellular technology started in Japan which resulted in the deployment of the first cellular networks in Tokyo. Within couple of years Nordic Mobile Telephony (NMT) started cellular operations in Europe. Along with it, systems such as Advanced Mobile Phone Service (AMPS) started in the USA, while Total Access Communication System (TACS) started in UK. These formed a part of what was called 'First Generation Mobile System which catered for speech services and were based on analogue transmission techniques. $1 \mathrm{G}$ is the first generation wireless telephone technology cell phones. They were analog cell phones and were introduced in 1980. It supported the $1 \mathrm{G}$ of analog cell phones speed up to $2.4 \mathrm{kbps}$. This cellular Phone technology works in frequency band of $150 \mathrm{KHz}$ (Garsha S.N and Ashna K; 2016).

The main drawback of First generation mobile networks is that the cellular networks were unable to interoperate between countries. IG has low capacity, unreliable handoff, poor voice links, no data communication and no security since voice calls were played back in radio towers making these calls susceptible to unwanted situation. Even an unknown receiver with all band radio can listen to the conversation. Thefts of airtime is also a major issue that has been reported in this area (Agrawal J; 2015). User has to enable a transmission button and disable reception and hence it become 'press to transmit' system (Mohammad M. M and Kumar S; 2015).

Beside the First Generation based on analogue, all the systems initially developed were quite incompatible with each other, facilities such as roaming within the continent were impossible and most countries had only one operator. The penetrator was low. Handsets were also expensive, the minimum being more than $\$ 1000$. First generation technology also had an inherent limitation in terms of channels (Mishra R. A; 2007).

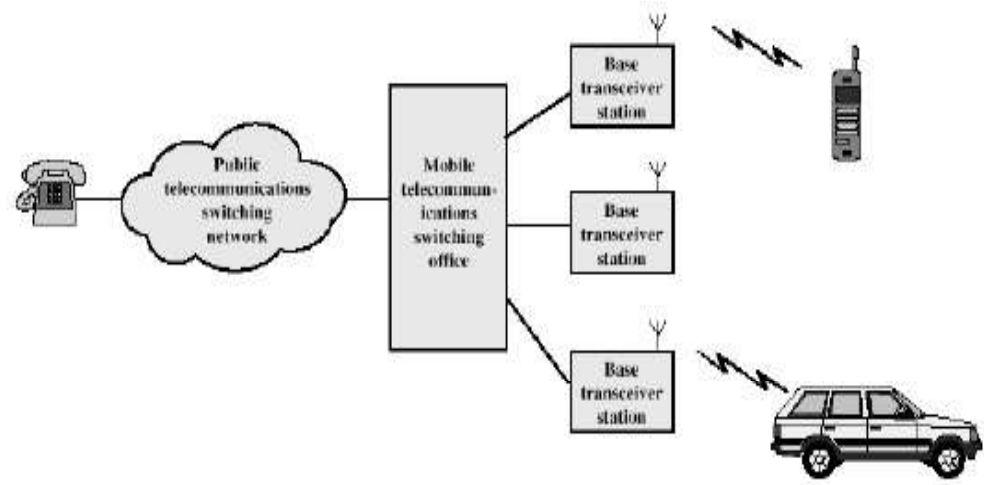

Figure 1: 1G Cellular Network Architecture

Source: http://www.sis.pitt.edu/ dtipper/tipper.html.

\subsection{Examples of First Generation Cellular Networks}

\subsubsection{Nordic Mobile Telephony (NMT)}

NMT was created in 1981 as a response to the increasing congestion and heavy requirement of the auto radio puhelin or car radio phone (ARP) mobile phone network. It is based on analogue technology and two variant exist namely NMT 450 and NMT 900. The numbers indicate the frequency bands used. The drawbacks of the original NMT specification is that traffic was not encrypted and simple scanner can be used to tune to correct frequency. Some scanners have had the NMT band deleted so they could not be accessed (Mishra R. A; 2007) 


\subsubsection{Advanced Mobile Phone System (AMPS)}

The first cellular licenses in the US were awarded in 1983 in Chicago and Baltimore-Washington area using the AMPS. The AMPS was based on the Frequency Division Multiple Access (FDMA) technology which allows multiple users in a cell or cell sector. This system did not only had capacity related problems but the securities system was poor. Efforts were made to address these problems, especially the capacity related ones but the results were not sufficient. The AMPS operates at frequency bands of $824-845 \mathrm{MHz}$ for uplink and $824-894 \mathrm{MHz}$ for downlink and is frequency modulated (FM) with channel bandwidth of $30 \mathrm{KHz}$ and signal peak at $1200 \mathrm{bps}$ (Rappaport T. S; 2002).

\subsection{Second Generation Wireless Networks (2G)}

$2 \mathrm{G}$ is the second-generation wireless cell phones, based on digital technologies and in early 1990s. In $19912 \mathrm{G}$ was launched in Finland. 2G provided services such as text message, picture messages and MMS. 2G has greater security for both sender and receiver. All text messages are digitally encrypted, which allows for the transfer of data in such a way that only intended receiver can receive and read it. $2 \mathrm{G}$ system uses digital mobile access technology such as TDMA and CDMA. TDMA divides signal in time slots while CDMA allocates each user a special code to communication over multiple physical channel. Different TDMA technologies are GSM, PDC, iDEN, iS-136. CDMA technology is IS-95. GSM was first 2G system. GSM (Group Special Mobile) has origin from Europe. GSM uses TDMA to multiplex up to eight calls per channel in the $900 \mathrm{MHz}$ and $1800 \mathrm{MHz}$ bands. GSM can't only deliver voice but also circuit switched data at speed up to $14.4 \mathrm{kbps}$. In between $2 \mathrm{G}$ and $3 \mathrm{G}$ there is another generation called 2.5G. 2.5G represent handset with data capabilities over GPRS but 2.5G has not brought out any revolution (Agrawal et al; 2015).

Second generation wireless system employ digital modulation (Digitalization) and advanced cell processing capacities (Rappaport T. S; 2002). Digitalization means that the sound of the speaker's voice was processed in a way that imitated a human ear through technique such as sampling and filtering. This made it possible for many more mobile users to be accommodated in the radio spectrum (Mishra R.A. 2007). The systems employ dedicated control channel within the air interface for simultaneously exchanging voice and control information between the subscriber, the base station, and the Mobile Switching Center (MSC) while a call is in progress (Rappaport T. S; 2002).

Second generation $(2 \mathrm{G})$ wireless networks were specially designed to provide paging, and other data services such as facsimile and high-data rate network access. The networks controlling structure is more distributed in second generation wireless systems, since mobile stations assume greater control functions. The mobile units in these networks perform several other functions not performed by $1 \mathrm{G}$ such as received power reporting, adjacent base station scanning, data encoding, and encryption. $2 \mathrm{G}$ systems have been designed to reduce the computational and switching burden at the base station or MSC, while providing more flexibility in the channel allocation scheme so that systems may be deployed rapidly and in a less coordinated manner (Rappaport T. S; 2002).

Some of the drawbacks of $2 \mathrm{G}$ digital system are drop outs under slightly worse conditions where a long system is static, another problem on $2 \mathrm{G}$ cellular system is that sometimes weaker digital system may not reach the cell tower and more so digital has a jagged steppy curve whereas analog has a smooth decay curve (Reinhardt Andy 2005). 2G system lacked capacity, Global roaming and quality not to mention amount of data that could be sent. This all led to the industry working on a system that had more global reach (Nilchil B et al; 2007).

\subsection{Examples of Second Generation Cellular Networks}

\subsubsection{Global System for Mobile Communication (GSM)}

GSM was first developed in the 1980s, from 1982 to 1985 GSM group discussions were held to decide between building an analogue or a digital system. After multiple field tests, it was decided to build a digital system and a narrow band TDMA solution was chosen. GSM was the first commercially operated digital cellular system with 
Radiolinja in Finland (Mishra R., 2007). The modulation scheme chosen was Gaussian Minimum Shift Keying (GMSK). The GSM system operates at various radio frequencies, with most of them operating at $900 \mathrm{MHz}$ with uplink frequency of $880-915 \mathrm{MHz}$ and downlink frequency of $925-960 \mathrm{MHz}$. Some also operates at $1800 \mathrm{MHz}$ with uplink frequency of $1710-1785 \mathrm{MHz}$ and downlink frequency of $1805-1880 \mathrm{MHz}$ with channel bandwidth of 200KHz. Services including voice mail, SMS, Call waiting were provided aside from making calls.. The cell radius in the GSM network varies depending upon the antenna height, antenna gains, and propagation conditions. In GSM network cells sizes are classified into macro, micro, pico and umbrella with macro cells being the biggest and pico and umbrella cells being the smallest (Mishra R. A; 2007). The key advantage of GSM systems has been higher digital voice quality and low cost alternatives to making calls such as text messaging. Equipment are deploy from different vendors because the open standard allows easy interoperability.

\subsubsection{Code Division Multiple Access (CDMA)}

CDMA-one (IS-95) (where IS stands for Interim Standard) is a second generation system, offering advantages such as increase in capacity (almost 10times that of the AMPS), improved quality and coverage, and also improved security system etc. Enhancement of the CDMA-one was IS-95B, also called 2.5G of CDMA technology which combined the standards IS-95A, ANSI-J-STD-008 and TSB-74. Major advantages of this system include frequency diversity, increase privacy, an interference limited system etc, while some disadvantages of this system include the air interface, which is the most complicated, soft hand-off, which is more complicated than the ones used in the TDMA/FDMA system (Mishra R. A; 2007).

\subsubsection{Digital Advanced Mobile Phone System (D-AMPS)}

IS-54 and IS-136 are the second generation mobile systems that constitute the D-AMPS. The D-AMPS used existing AMPS channels and allows for smooth transition between digital and analogue systems in the same area. Capacity was increased over the preceding analogue design by dividing each $30 \mathrm{KHz}$ channel pair into three time slots and digitally compressing the voice data, yielding three times the call capacity in a single cell. DAMPS used the $800 \mathrm{MHz}$ and $900 \mathrm{MHz}$ frequency bands but each $30 \mathrm{KHz}$ channel (created by FDMA) is further divided into three TDMA, which triples the channels available and the number of calls (Mishra R. A; 2007).

\subsubsection{General Packet Radio Service (GPRS)}

GPRS is a non-voice i.e. data value added service to the GSM network. This is done by overlaying a packet based air interface on the existing circuit switched GSM network. The voice traffic is circuit switched while data traffic is packet switched. Packet switching enables the resources to be used only when the subscriber is actually sending and receiving the data. The amount of data that can be transferred is dependent upon the number of users. Theoretical maximum speeds of up to $171.2 \mathrm{Kbps}$ are achievable with GPRS. GPRS allows for interconnection between the network and the internet. However there some limitations in the GPRS network, such as low speed (Mishra R. A; 2007).

\subsubsection{Enhanced Data Rate for GSM Evolution (EDGE)}

The limitation of the GPRS network was eliminated to a certain extent by the introduction of the EDGE technology. It is considered to be a subset of the GPRS as it can be installed in any system that has GPRS deploy on it. EDGE is also called 2.75G and can transfer data at the rate of 9.6-200kbps. However, the major advantage is that existing GSM networks can be upgraded for the same, therefore preventing huge costs needed to rollout new network technology. General features of EDGE include enhanced throughout per time slot (8.8$59.2 \mathrm{kbps} /$ timeslot), modulation changes from GSMK to 8-PSK, decreased sensitivity to the 8-PSK signal and coverage. 


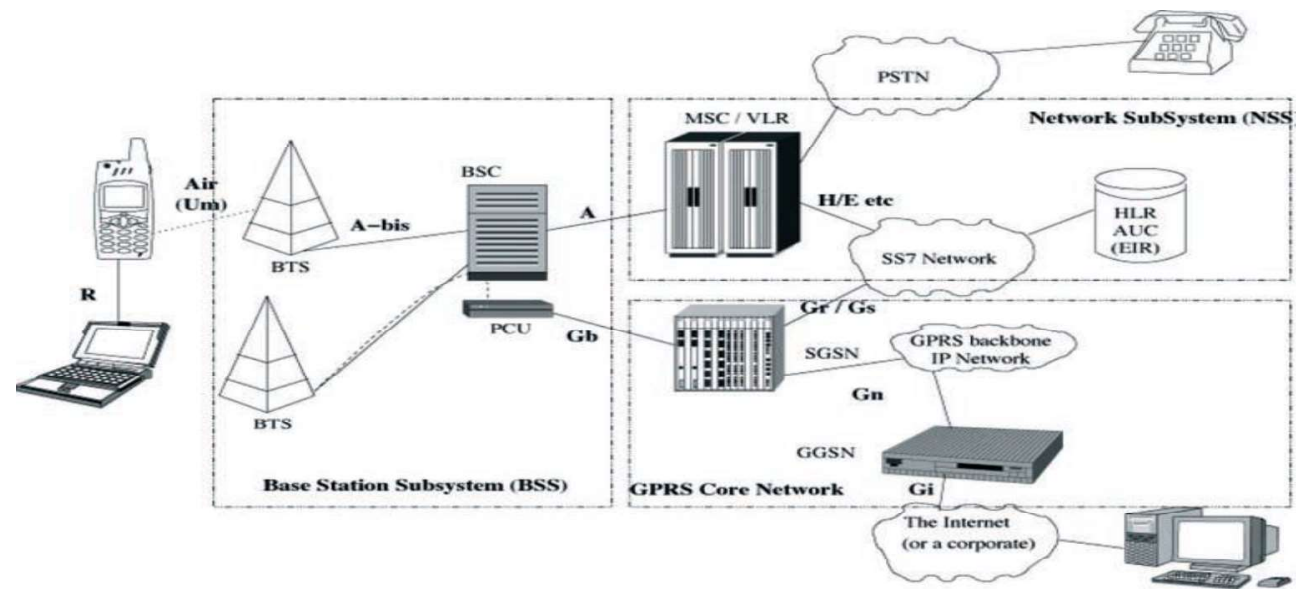

Figure 2: GSM and GPRS System Architecture

Source: Ajay R Mishra; 2007.

\subsubsection{Benefits of 2G over Its Predecessor}

$2 \mathrm{G}$ allows far greater phone penetration hence has more efficient spectrum, $2 \mathrm{G}$ cellular network is digitally encrypted and SMS text messaging and other services were started with $2 \mathrm{G}$ cellular network.

\subsection{Third Generation Cellular Networks (3G)}

The third generation cellular networks were developed with the aim of offering high speed data and multimedia connectivity to subscribers. $3 \mathrm{G}$ is the third generation of mobile phone and technology. Third generation wireless systems evolved from matured second generation systems. The aim of third generation wireless networks is to provide a single set of standards that can meet a wide range of wireless applications and provide universal access throughout the world. In third generation wireless systems, the distributions between cordless telephones and cellular telephones disappeared and a universal personal communicator (a personal handset) provide access to a variety of voice, data, and video communication services and operates in varied region (dense or sparely populated regions) and serve both stationary users and vehicular users traveling at high speeds (Rappaport T. S; 2002). The frequency spectrum of $3 \mathrm{G}$ transmissions is $1900-2025 \mathrm{MHz}$ and $2110-2200 \mathrm{MHz}$. The satellite service uses 1980-2010-2025MHz and 2110-2170MHz bands for terrestrial UTMS. (Henten A., and Saugstrup, D 2004).

One of the main characteristics of $3 \mathrm{G}$ is to provide mobile multimedia services at a transmission rate of $144 \mathrm{kbps}$ indoors (Mohr W, and Konhauser, W. 2000). 3G basically include the data services and the voice services. Data services are further categorized into Mobile Internet Access, customized Infotainment, multimedia services and location based services. The voice services include Rich voice and personalizing calls (Nauman Aftab and Ammar Igbal 2006). 3G is being used as broadband integrated services digital networks such as the internet and other public and private databases (Chevillat, P.R., 2003). 3G technologies enable network operators to offer users a wider range of more advanced services while achieving greater network capacity through improved spectral efficiency (Holma, H and Toskala A; 1997). Services include wide area wireless voice telephony, video calls, broadband wireless data mobile television, GPS and video conferencing, and all in a mobile environment (Mishra R. A; 2007).

\subsubsection{Examples of Third Generation Cellular Networks}

\subsubsection{CDMA 2000}

CDMA2000 has variants such as 1X, 1XEV-DO, 1XCV-DV and 3X. It is also known as the High Rate Packet Data Air Interface Specification. It delivers $3 \mathrm{G}$ like services up to $140 \mathrm{Kbps}$ peak rate while occupying a very 
small amount of spectrum $(1.25 \mathrm{MHz}$ per carrier). $1 \mathrm{XEV}-\mathrm{DO}$ phase one is an enhancement that put voice and data on separate channels in order to provide data delivery at $2.4 \mathrm{Mbps}$ while EV-DV, or $1 \mathrm{XEV}$ phase two data speeds ranging from 3Mbps to 5Mbps (Mishra R. A; 2007).

\subsubsection{Universal Mobile Telecommunication System (UMTS)}

UMTS is one of the third generation (3G) mobile phone technologies. It was first developed in year 2000. It uses W-CDMA as the underlying standard. The modulation scheme chosen was QPSK. The ITU eventually accepted W-CDMA as part of the IMT-2000 family of 3G standards. W-CDMA was selected as the air interface for UTMS, the $3 \mathrm{G}$ successor to GSM. Some key features include the support to two basic modes FDD and TDD, variable transmission rates, inter-cell asynchronous operation, adaptive power control, increased coverage and capacity etc. W-CDMA is merely the air interface as per the definition of IMT-2000, while UTMS is a complete stack of communication protocols designated for $3 \mathrm{G}$ global mobile telecommunications. UMTS uses a pair of $5 \mathrm{MHz}$ channels, one in the $1900 \mathrm{MHz}$ range for uplink and one in the $2100 \mathrm{MHz}$ range for downlink. The specific frequency band defined by the UTMS standard are $1885-2025 \mathrm{MHz}$ for uplink and $2110-2200 \mathrm{MHz}$ for downlink (Mishra R. A; 2007).

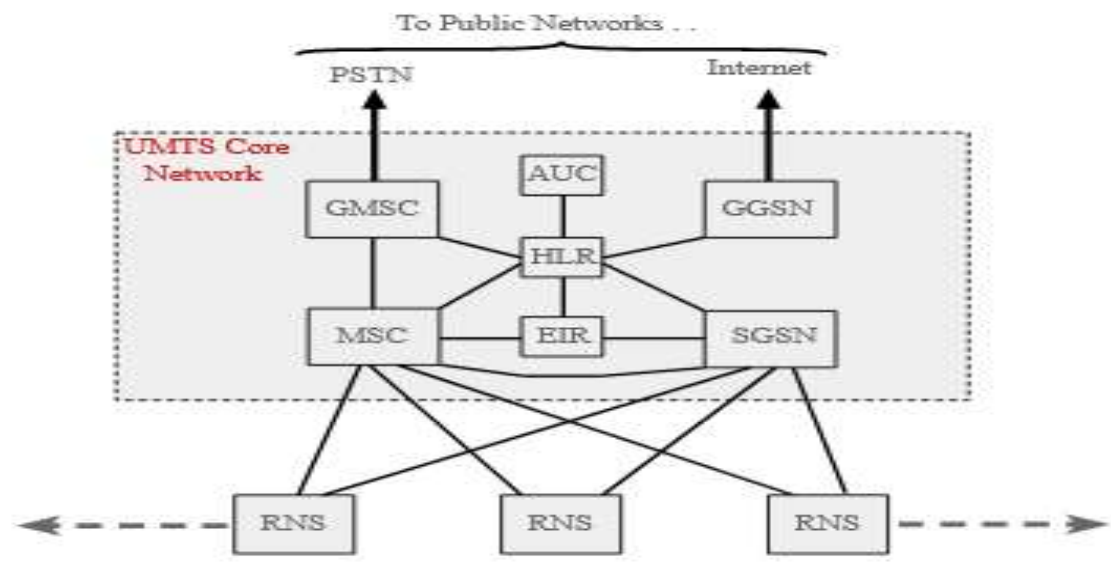

Figure 3: UMTS Network Architecture.

Source:https:/www.electronics-notes.com/images/umts-network-architecture-01.svg

\subsubsection{High Speed Download Packet Access (HSDPA) in UMTS}

High Speed Downlink Packet Access (HSDPA) is a packet based data service in the downlink having a transmission rate up to $8-10 \mathrm{Mbps}$ over the $5 \mathrm{MHz}$ bandwidth. Implementation of this technique allow data speed to increase to almost five times that of the most advanced Wideband Code Division Multiple Access (WCDMA) networks. Also, the base station capacity increased by double (Heikki K; 2006). The system capacity and the user data rates are increased by implementation of HSDPA mainly intended for non-real-time traffic, but can also be used for traffic with tighter delay requirements (Mishra R. A; 2007).

\subsubsection{Benefits of 3G over Its Predecessors}

3G has several benefits over its predecessors such as several times higher data speed, enhanced audio and video streaming, video-conference support, web and WAP browsing at higher speeds and IPTU (TV through the internet) support etc. (Mishra R.A 2007). 


\subsection{Fourth Generation Mobile Networks}

The previous generation of mobile network had their merits and demerits but none of them had to completely replace the other. Even IMT-2000 was not able to break the bottle neck of high data rate and capacity, this led to the formation of a new generation, referred to as beyond $3 \mathrm{G}$ generation or $4 \mathrm{G}$. 4G has being in existence since 2010 and its otherwise known as Long Term Evolution Network (LTE). LTE evolves from the third generation technology which is based on WCDMA and defines the long-term evolution of the 3GPP UMTS/HSPA cellular technology. The modulation scheme chosen for 4G are QPSK, QAM and 64QAM. It offers high data rates, lower latency and greater spectral efficiency, high voice quality, easily access internet, streaming media, video calling, simple protocol architecture and efficient multicast/broadcast than its predecessors (Tchao, et al., 2008). Instead of developing new radio interfaces and new technology existing and newly developed wireless system like the GPRS, EDGE, Bluetooth, WLAN and Hiper-LAN were integrated together. In 2008, the ITU-R (International Telecommunication Union) organization specified the IMT-Advanced requirements for 4G standards, setting peak speed requirements for $4 \mathrm{G}$ service at $100 \mathrm{Mbps}$ for high mobility communication (such as from trains and cars) and 1Gbps for low mobility communication (such as pedestrians and stationary users). 4G has a download speed of $67.65 \mathrm{Mbps}$ and upload speed of $29.37 \mathrm{Mbps}$ which is three times faster speeds which makes load quicker, and this makes stream videos and Podcasts possible without first waiting for them to buffer. The 4G operates at frequency bands of $2500-2570 \mathrm{MHz}$ for uplink and $2620-2690 \mathrm{MHz}$ for downlink with channel bandwidth of 1.25-20MHz (Noorus Sasah; 2016).

4G is a combination of existing and advanced technologies with seamless handover between technologies irrespective of the geographical location, having high data rates, high capacity, and high quality of service and low cost. The 4G networks hierarchy consist of four broad levels of networks namely; the personal networks, the local networks, the cellular networks and the satellite based networks. $4 \mathrm{G}$ can perform vertical handovers and horizontal handovers. $4 \mathrm{G}$ user devices is able to work with different wireless technologies and also able to select the appropriate technology to make use of a particular service by performing handovers (Mishra R. A; 2007).

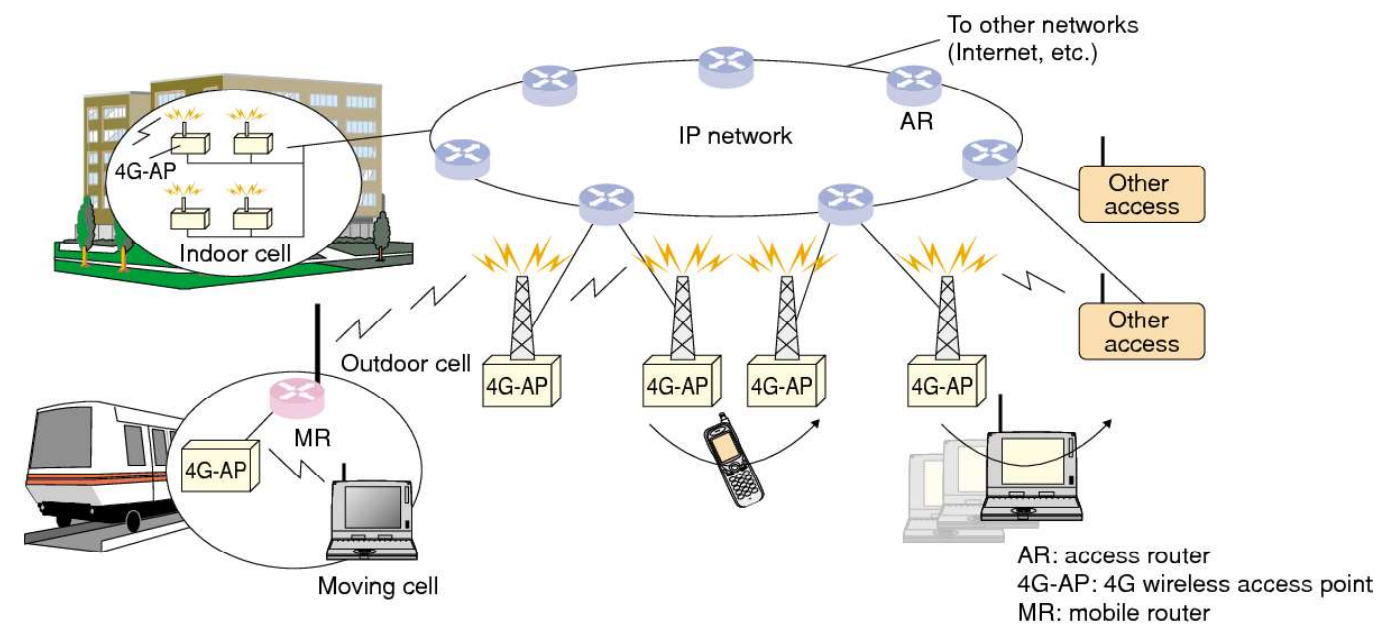

Figure 4: 4G Cellular Network Architecture.

Source:https://www.google.com.ng/url?sa=i\&url=https $\% 3 \mathrm{~A} \% 2 \mathrm{~F} \% 2 \mathrm{Fwww}$. semanticscholar.org\%2Fpaper\%2FO verview-of-the-Fourth-generation

\subsection{Key Technology of $4 G$}

Some of the key technologies of $4 \mathrm{G}$ are Orthogonal Frequency Division Multiplexing (OFDM), Software defined Radio (SDR) and Multiple-Input Multiple-Output which uses Signal multiplexing between multiple transmitting antennas space and time or frequency. It is well suited to OFDM, as it is possible to process independent time symbols as soon as the OFDM waveform is correctly designed for the channel. More also, handover technologies based on mobile IP technology have been considered for data and voice. 


\subsection{Applications of 4G Networks}

4G provides user services at all times, even if the user is off-site. Its provides users with virtual navigation through which a user can access a data-base of the street, buildings etc. 4G is used in Tele-Geoprocessing applications which is a combination of GIS (Geographical Information System) and GPS (Global Positioning System) in which a user can get the location by querying. 4G is also applicable in Tele-Medicine and Education where it support remote health monitoring of patients and for people who are interested in lifelong education, $4 \mathrm{G}$ provides a good opportunity. Natural disasters can cause breakdown in communication systems and $4 \mathrm{G}$ is expected to restore such crisis issues in a few hours (Omar Nader et al; 2015).

\subsection{Benefits of 4G over Its Predecessors}

4G benefits are higher bandwidth, lower cost of networks and equipment, the use of license exempt spectrum, higher capacity and quality of service enhancement and higher revenue. $4 \mathrm{G}$ networks enhance users access to broadband multimedia services with lower cost where mostly needed, and inter network roaming.

\subsection{Challenges and Limitations of 4G Networks}

The current software radio technology is not completely feasible for all wireless networks due to the fact that it is impossible to have just one antenna and one LNA to serve the wide range of frequency bands. Also the existing analogue to digital converters (ADC) used in mobile stations are not fast enough. And in order to allow realtime execution of software implemented radio interface functions, such as frequency conversion, digital filtering, spreading and de-spreading, parallel DSPs have to be used, thereby increasing the circuit complexity and high power consumption and dissipation. Supporting quality of service in 4Gnetworks is a major challenge due to varying bit rates, channel characteristics, bandwidth allocation, fault-tolerance levels and hand-off support among heterogeneous wireless networks (Mishra R. A; 2007). Higher data prices for consumers, very expensive and hard to implement are the basic disadvantages of 4G (Jyotsna Agrawal et al; 2015).

\subsection{Fifth Generation Cellular Mobile Technology}

Since 2012, Telecommunication Standards development organizations (SDO), with the help of their industry partners, have been researching ways to improve mobile communication networks, link people, devices and data through a smart network, and enable a seamlessly connected society. Fifth Generation (5G) Mobile technology represent the next iteration of mobile communication technologies that were designed to improve current $4 \mathrm{G}$ mobile networks. In 2018, telecommunication providers began deploying fifth-generation (5G) networks to meet growing demands for data from consumer and industrial users e.g (video streaming, virtual reality applications, support new industrial uses) e.g. (industrial sensors, industrial monitoring system), perform advanced data analytics, and enable the use of advanced technologies (smart city applications, autonomous vehicles and navigation). $5 \mathrm{G}$ networks become operational in some developed countries such as China, US and UK early this year $2020.5 \mathrm{G}$ networks operate at the frequency spectrum of $3 \mathrm{GHz}$ to $300 \mathrm{GHz}$ with bandwidth of $0.25-1 \mathrm{Gbps}$. $5 \mathrm{G}$ supports high performance mobility of up to $500 \mathrm{~km} / \mathrm{h}$, data rate of up to $20 \mathrm{Gbps}$, spectral frequency of $30 \mathrm{bps} / \mathrm{Hz}$, U-Plane latency of $0.5 \mathrm{~ms}$ and C-Plane latency of $10 \mathrm{~ms}$. $5 \mathrm{G}$ networks are expected to provide faster speeds, greater capacity, and the potential to support new features and services (Jill G. Gallagher and Michael E. Devine 2019). 5G networks offer the increased bandwidth, constant connectivity, and low latency services which can enhance and expand the use of mobile technologies for consumers and businesses (David Talbot 2016). 5G is expected to yield significant and economic benefits. Market analyst estimated that in the United states, 5G could create up to 3million new jobs and add \$500 billion to the nation's gross domestic product (GDP) globally, analysts estimate that $5 \mathrm{G}$ technologies could generate $\$ 12.3$ trillion in sales activity across multiple industries and support 22 million job by 2035 (J. Stanley 2018).

There are several factors affecting all 5G deployments, including international decisions on standards and spectrum (e.g. auctioning spectrum reconfiguring users to accommodate $5 \mathrm{G}$, establishing agreement to share spectrum), the availability of $5 \mathrm{G}$ equipment and services. $5 \mathrm{G}$ requires thousand times higher mobile data as 
compared to $4 \mathrm{G}$. $5 \mathrm{G}$ user data-rate is greater than $1 \mathrm{Gbps}$. $5 \mathrm{G}$ also requires ten to hundred times numbers of connected devices as compare to $4 \mathrm{G}$. More battery life and five times reduced latency as compared to $4 \mathrm{G}$ are part of technical requirements of 5G (Arun Kumar and Mishra Gupta 2018).

Vision of $5 \mathrm{G}$ mobile communication are $100 \mathrm{X}$ network energy efficiency, Area traffic capacity of $10 \mathrm{Mbits} / \mathrm{sq} . \mathrm{metre}, 1 \mathrm{million}$ number connected devices per sqkm, $3 \mathrm{X}$ spectrum efficiency, $1 \mathrm{~ms}$ end to end round trip delay latency and 1-10Gbps connection to end point in the field (Guangyi Liu and Dajie Jang 2016). 5G bandwidth is $1000 \mathrm{xBW}$ per unit area and the technology is wwww. 5G applications are Voice, Data, Video call, UHD videos, V2X, 10T, Smart City/Factory/Home, Telemedicine, and wearable devices. 5G has a lot of features such as high response, high capacity, wider range of applications, connectivity speed up to $25 \mathrm{Mbps}$, more software option to upgrade, large broadcasting, less traffic, bi-directional and Support virtual private network (Rajat Verma 2018).

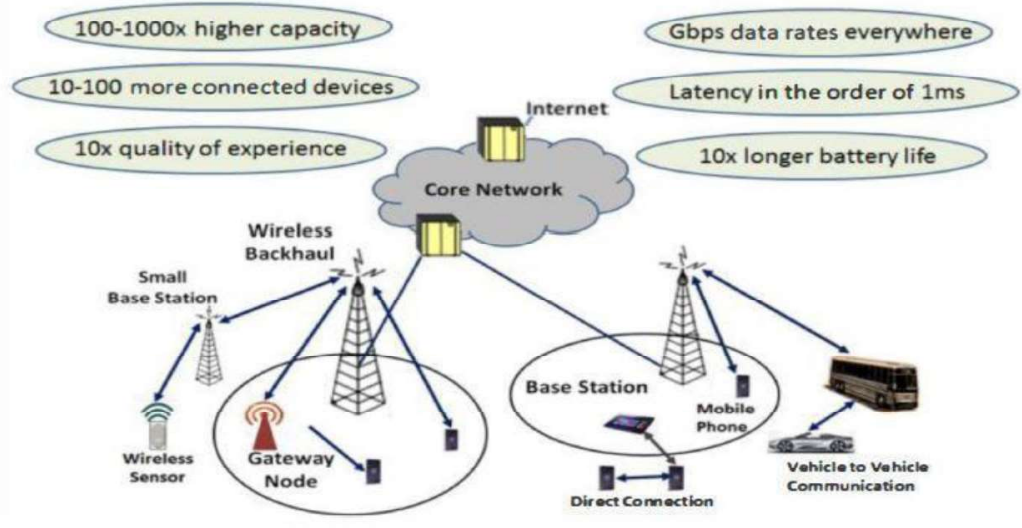

Figure 5: 5G Cellular Network Architecture

Source: Vrushalid Jorwar; 2016

\subsection{Key Technologies in $5 G$}

The key technologies in 5G are (a) Orthogonal Frequency Division Multiplexing (OFDM) where bandwidth is divided into number of subcarriers which are orthogonal to each other. It is implemented by using Fast Fourier Transform (FFT), Inverse Fourier Transform (FFT) and Inverse Fast Fourier Transform (IFFT) which has advantage of increase in data-rate, high capacity, and immune to inter symbol interference. (b) Orthogonal Frequency Band Multicarrier (OFBMC) which results in better utilization of available bandwidth and provide more robustness to the system. In this technique like OFDM, the available bandwidth is divided into number of subcarrier which are orthogonal with each another and further does not use cyclic prefix. (c) Universal Filter Multi Carrier (UFMC) which is based on a filter bank multicarrier and OFDM is considered to be best and novel modulation scheme for $5 \mathrm{G}$. The technique efficiently reduces the side lobe which increases the performance of the system. (d) Filter Band Multicarrier (FBMC) which an advanced technique of OFDM and does not use CP but uses arrays of filters at transmitter and receiver. The advantage of this technique is that without using the $\mathrm{CP}$, it can give an efficient and better performance than OFDM. (e) Generalized Frequency Domain Multiplexing (GFDM) which is non-orthogonal in nature and is first contender of non orthogonal waveform for $5 \mathrm{G}$ mobile communication system. In this technique, the modulation is based on a Balian low theorem and transmission is achieved by using a time and frequency localized pulse. (f) Cognitive radio which is an intelligent communication system that is aware of its surrounding environment and utilizes the methodology of understanding the environment and takes the decision (Arun Kumar and Mishra Gupta 2018). (g) Faster than Nygist rate increase the capacity by sending more data in the time domain. It utilizes linear model techniques 
where a pulse is sent at a faster rate in a time domain which results in ISI, loss of orthogonality but still the signal can be recovered by employing an advanced detection techniques.

\subsection{Benefits of 5G over Its Predecessors}

5G techniques is more than increasing maximum throughput, it include other benefits such as lower battery consumption, lower outage probability, high resolution and bi-directional large bandwidth shaping, easily manageable with the previous generations, better coverage and high data rates available at cell edge, multiple concurrent data transfer for paths, around $1 \mathrm{Gbps}$ data rate in mobility, more secure, better cognitive radio, spectral efficiency (Vrushali D. Jorwar; 2016). 5G technology is to facilitate subscriber supervision tools for the quick action. It is a new technology that will provide all the possible applications, by using only one universal device, and interconnecting most of the already existing communication infrastructures. 5G is most likely to provide huge broadcasting data (in Gigabit), which will support more than 60,000 connections. 5G is technological sound to support heterogeneous services (including private network) and possible to provide uniform, uninterrupted, and consistent connectivity across the world (Anwar M. Mousa; 2012); (Charu and Rajeev Gupta 2015) and (Garsha S.N and Ashua K. 2016).

\subsection{Challenges and Limitations of 5G Networks}

No technology is complete in itself and this is also the case with 5G. There are foreseen technical challenges and limitations of 5G such as inter-cell efficient Medium Access Control and Traffic Management, Legislation, multiple services, security, communications and high cost of infrastructure.

\subsection{Sixth Generation Mobile Network (6G)}

Several 6G activities have been commenced globally by industrial organizations and governments where objectives includes formulating and defining 6G technology and then re-adjusting the outline in addition to the business model of wireless systems. The united states FCC has proposed allocating the $95 \mathrm{GHz}$ to $3 \mathrm{THz}$ spectrum to be used for $6 \mathrm{G}$ research thereby setting the US as a pacesetter in the $6 \mathrm{G}$ Research race. $6 \mathrm{G}$ communications are expected to raise the bar currently set by $5 \mathrm{G}$ communications with the provision of enhanced services from the perspectives of network data availability mobile data rate, and seamless ubiquitous connection. $6 \mathrm{G}$ will usher in the same reliability as wired networks with a low error-rate considering the type of applications that will be supported. A host of futuristic currently non-existent wireless communication scenarios of the 2030s are predicted. $6 \mathrm{G}$ spectrum is very broad with frequency bands of $73 \mathrm{GHz}, 140 \mathrm{GHz}$ and $1-10 \mathrm{THz}, 6 \mathrm{G}$ will support performance mobility of up to $1000 \mathrm{~km} / \mathrm{h}$, Data rate $>1 \mathrm{Tbps}$, spectral efficiency of $100 \mathrm{bps} / \mathrm{Hz}$, U-plane latency $<0.1 \mathrm{~ms}$ and C-plane latency $<1 \mathrm{~ms}$ (Aggarwal S. and Kumar N 2019). The 6G system is expected to witness an unparalleled revolution that would significantly distinguish it from the existing generations and will drastically re-shape the wireless evolution from "connected thing to connected intelligence (Yastrebova A. et al., 2018); (Rappaport T.S et al., 2019); and (Yang P. et al., 2019). 6G will bring emerging technologies such as artificial intelligence, terahertz communications, optical wireless technology, free space optic network, block chain, three dimensional networking, quantum communication, unmanned aerial vehicle, cell-free communications, integration of wireless information and energy transfer, integration of sensing and communication, integration of access-backhaul networks, dynamic network slicing, holographic beam forming, and big data analytics that can assist the 6G architecture in guaranteeing the quality of service (Wang H. et al., 2014) and (Yang P et al., 2019).

The proposed 6G will have key features such as: (a) Multiband ultrafast speed transmission in $\mathrm{THz}$ band $(0.1$ $10 \mathrm{THz}$ ); (b) Operate at light frequency band (c) Energy efficient communication e.g. Harvest energy from ambient radio-frequency signals, sunlight and wireless power charging (e.g. inductive coupling, magnetic resonance coupling, microwave radiation and distributed laser charging) (d) Artificial Intelligence e.g. operational intelligence, Environmental Intelligence, service intelligence. (e) High security, secrecy and privacy e.g. well- integrated multilevel security like distributed management and mechanism and (f) Quantum key distribution via VLC and block chain technology (Mohammed et al., 2020); (Sengupta K. et al., 2018). 
6G will have very many applications such as Voice, Data, Video call, video chat, High definition TV, Three dimensional integrated communications, Tactile/Haptic internet, full -sensory digital sensing and reality, fully automated driving, Industrial internet, and internet of bio-nanothings

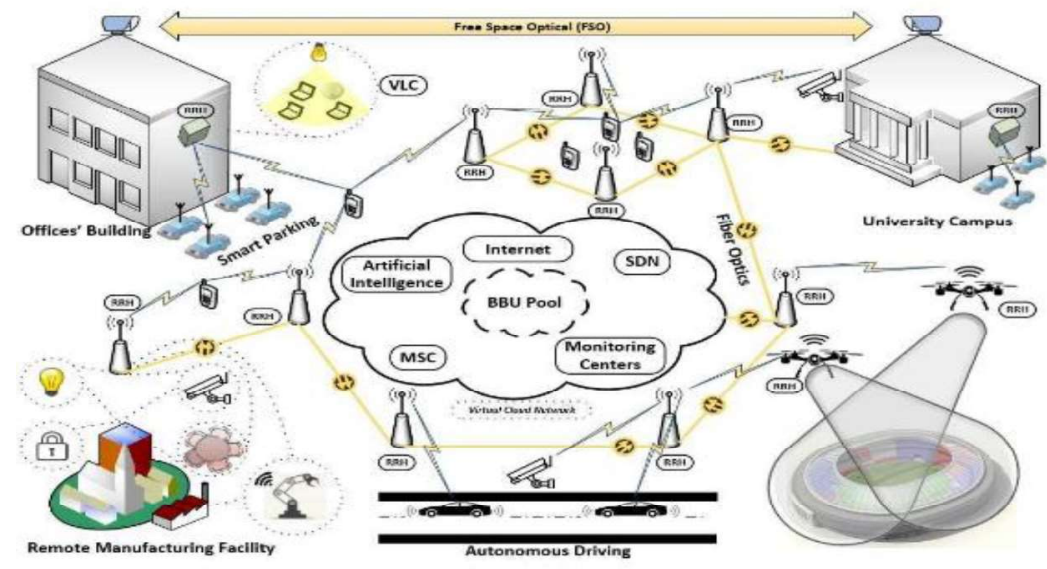

Figure 6: Proposed 6G Cellular Network Architecture

Source: https://www.rfwireless-world.com/Technology/

\subsection{Expected Benefits of 6G over Its Predecessors}

$6 \mathrm{G}$ technology is expected to come with a lots of benefits including ultra fast to access Internet, data rates up to 10-11Gbps, Home automation and other related applications, Smart Homes, Cities and villages, use in the production of energy from galatic world, Space technology and defense applications will be modified with $6 \mathrm{G}$ networks, Home based ATM systems, Satellite to satellite communication for the development of mankind, Natural calamities will be controlled with $6 \mathrm{G}$ networks and Sea to space communication will be possible with 6G (Mohammed et al., 2020); (Sengupta K. et al., 2018).

\subsection{Conclusion}

The past few decades have witnessed tremendous growth in cellular network technology and industry. Each generation of cellular network comes with handful benefits and improved architecture to meet the demand of end users. The challenges and limitations of one generation usually lead to evolution of another generation with additional key features aimed at improving technology, achieving greater efficiency and multiple applications. The competing deployment of $5 \mathrm{G}$ technologies among countries like China, USA, South Korea and UK is aimed at capturing the bulk of the economic benefits of the new technology as it is loaded with features and applications that will meet users and industrial demands than its predecessors. 5G technology unlike its predecessors is going to support heterogeneous services (including private network) and provide uniform, uninterrupted, and consistent connectivity across the world with huge broadcasting data (in Gigabit), which will support more than 60,000 connections. The envisioned $6 \mathrm{G}$ system is expected to witness an unparalleled revolution that would significantly distinguish it from the existing generations and will drastically re-shape the wireless evolution from connected thing to connected intelligence through its seamless features. Conclusively, the world will continue to witness evolution of new cellular networks with advanced technology, seamless features and amazing applications

\section{References}

Agrawal, J, Patel, R., Mor P., Dubey P., Keller J. M 2015, "Evolution of Mobile Communication Network from $1 \mathrm{G}$ to 4G.International Journal of Multidisciplinary and Current Research Vol. 3, no 11\&12. 
Aggarwal S; Kumar, N. Fog computing for 5G-Enabled Tactile Internet Research Issues, Challenges, and Future Research Directions. Mob. Network. April. 2019, 1-28.

First Generation Cellular Telecommunications and Networking, http://www .sis. pitt.edu/ $\sim$ d tipper .html downloaded on 15/06/2020.

Garsha Sai Nitesh and Ashna Kakkar 2016, Generations of Mobile Communication. International Journal of Advanced Research in Computer Science and Software Engineering Vol.6 Issue 3, ISSN: 2277128X Page 320-324.

Generation of Network 1G, 2G, 3G, 4G, 5G. Slideshare.net.1st 6G wireless submit. Available online: http://www.worldtimezone.com/5G.html (accessed on May 112020.

Henten A., Saugstrup, D. 2004, 3G Standards: The battle between WCDMA and CDMA2000, http://www.cict.dtu.dk/publications/working papers/ctiwp90.pdf.

H. Wang; W. Wang; X. Chen and Z. Zhang wireless information and energy transfer in interference aware massive MIMO Systems in prac. IEEE Global Conference, Austin, TX, 2014, page 2556-2561.

Holma, H., Toskala, A. "WCDMA for UMTS-HSPA evolution and LTE".Ed. Wiley.

https://www.nibusinessinfo.co.uk/advantages-and-disadvantages-mobile-technology.

https://www.tutorialspoint.com 5G Advantages and Disadvantages.

https://www.rfwireless-world.com/Technology/

https://www.electronics-notes.com/images/umts-network-architecture-01.svg.

ITU-R PDNR WP8F. Vision, framework and overall objectives of the future development of IMT-2000 system beyond IMT-2000, 2002

Nauman Aftab and Ammar Igbal 2006, 3G Mobile Communication Services Perspective, Master thesis, continuation courses.

Nilchil Bhandari, Shivinder Deura and Karamdeep Singh, Evolution of Cellular Network. From 1G to 5G, International Journal of Engineering and Techniques Vol. 3 issue 5, 2007.

Mishra, Ajay K. " Fundamental of Cellular Network Planning and Optimization 2G/2.5G/3G.....Evolution of 4G, John Wiley and Sons, 2004.

Mohammad Meraj ud in Mir, and Sumit Kumar 2015; Evolution of Mobile Wireless Technology from 0G to 5G; International Journal of Computer Science and Information Technologies vol. 6(3). Page 2545-2551.

Mohammed H. Alsharif, Anabi Hilary Kelechi, Mahmoud A. Albreem Shehzad Ashraf Chaudhry, M. Sultan Zia and Sunghwan Kin, Six Generation (6G) Wireless Networks: Vision, Research Activities, Challenges and Potential Solutions. 2020 symmetry review

P. Yang; Y. Xiao, M. Xiao, and S, Li, 6G wireless communications vision and potential techniques "IEEE Network. Vol. 33, no: 4, page 70-75 July/ August 2019.

Richard K.W 2000, UMTS overview, Electronics and Communication Engineering Journal. Reinhardt Andy, "A Telecom show's No 'Gloom". Businesses February 22, 2005.

Rappaport, T. S., Xing, Y., Kauhere O. S; Madanayake A; Mandal S; Trichopoulos, G.C wireless communications and application above 100GHz: Opportunities and Challenges for 6G and beyond IEEE Access 2019, 7, 78729-78757 [CrossRef].

Sengupta, K; Nagatsuma T; Mittleman D.M Tetrahertz integrated electronic and hybrid electronic-Photonic Systems. Nat. Electron. 2018, 622-635 [CrossRef]. 
Universal Mobile Telecommunication Systems (UMTS), Requirements for the UMTS Terrestrial Radio Access System (UTRA), ETSI Technical Report, UMTS 21.01 version 3.0.1, 1997.

UMTS world 2009 "UMTS/3G History and future milestone"[online]. Available: http://www. umtsworld.com/units/history.htm.

Vrushalid Jorwar 2016; 5th Generation Mobile Network, International Journal of Advanced Research in Computer Engineering and Technology Volume 5, Issue 6, Page 1716.

Yastrebova, A; Kirichek, R; Koucheryavy Y; Borodin A; Koucheryavy A; Future networks 2030: Architecture and requirement. In proceedings of the 2018 10th International Congress on Ultra- Modern Telecommunications and Control Systems and Workshops (ICUMT), Moscow, Russia, 5-9 Nov.2018; Page 1-8 